\title{
SOME DISTORTION THEOREMS FOR NEW SUBCLASS OF HARMONIC UNIVALENT FUNCTIONS
}

\author{
MOHAMMAD MEHDI SHABANI ${ }^{1}$, SAEED HASHEMI SABABE $^{2 *}$ \\ 1 Department of Mathematics, University of Shahrood, Shahrood, Iran. \\ 2 Young Researchers and Elite Club, Malard Branch, Islamic Azad \\ University, Malard, Iran.
}

\begin{abstract}
In the present paper, we introduced and study a new class of harmonic univalent functions on unit disc $\mathbb{U}$. also we obtain coefficient conditions, extreme points, convolution condition for the above class of harmonic univalent functions.
\end{abstract}

\section{INTRODUCTION}

A continuous function $f=u+i v$ is a complex-valued harmonic function in a complex domain $\mathbb{C}$ if both $u$ and $v$ are real harmonic. In any simply connected domain $B \subset \mathbb{C}$, we can write $f=h+g$, where $h$ and $g$ are analytic in $B$. We call $h$ and $g$ are analytic part and co-analytic part of $\mathrm{f}$ respectively. Clunie and Sheil-Small [5] observed that a necessary and sufficient condition for the harmonic functions $f=h+g$ to be locally univalent and sensepreserving in $B$ is that $\left|h^{\prime}(z)\right|>\left|g^{\prime}(z)\right|$ for all $z \in B$. Denote by $\mathcal{S}_{H}$ the class of functions $f=h+\bar{g}$ that are harmonic univalent and sense-preserving in the unit disk $\mathbb{U}=\{z \in \mathbb{C}:|z|<1\}$ for which $f(0)=f_{z}(0)-1=0$.

In 1984, Clunie and Sheil-Small [5] investigated the class $\mathcal{S}_{H}$ as well as its geometric subclasses and obtained some coefficient bounds. They proved that although $\mathcal{S}_{H}$ is not compact, it is normal with respect to the topology of uniform convergence on compact subsets of $\mathbb{U}$. Meanwhile the subclass $\mathcal{S}_{H}^{0}$ of $\mathcal{S}_{H}$ consisting of the functions having the property $f_{\bar{z}}(0)=0$ is compact.

Some of the following subcalsses has been studied as mentioned:

(i) The classes $\mathcal{S}_{H}(1,0 ; 0,0)=\mathcal{S}_{H}$ and $\mathcal{S}_{H}(2,1 ; 0,0)=\mathcal{C}_{H}$ were studied by Avci and Zlotkiewicz in [4].

(ii) The classes $\mathcal{S}_{H}(1,0 ; \alpha, 0)=\mathcal{S}_{H}(\alpha)$ and $\mathcal{S}_{H}(2,1 ; \alpha, 0)=\mathcal{C}_{H}(\alpha)$ were studied by Ozturk and Yalcin in [9].

E-mail address: Mohammadmehdishabani@ymail.com, Hashemi_1365@yahoo.com. 2000 Mathematics Subject Classification. 30C45; 30C50.

Key words and phrases. Convex harmonic functions, Starlike harmonic functions, Univalent harmonic functions, Extremal problems, differential operator.

* Corresponding Author, ORCID: 0000-0003-1167-5006. 
(iii) The class $\mathcal{S}_{H}(m, n ; \alpha, 0)=\mathcal{S}_{H}(m, n, \alpha)$ was studied by Dixit et al. in [6].

(iv) The class $\mathcal{S}_{H}(1,0 ; \alpha, \beta)=\mathcal{S}_{H}(\alpha, \beta)$ was studied by Seoudy in [12].

(v) The class $\mathcal{S}_{H}(n+1, n ; \alpha, 0)=\mathcal{S}_{H}(\alpha, n)$ was studied by Aouf et al. in $[11]$.

In secion 2, we denote some fundamental defenitions, theorems and lemmas and in section 3 , we investigate several properties of the classes $\mathcal{S}_{H}(m, n ; \alpha, \beta)$ and $\mathcal{S}_{H}^{0}(m, n ; \alpha, \beta)$. Also, we generalize, improve and correct some results of Ozturk and Yalcin [9]. More recent works in this area can be found in [1] and [2].

\section{Prilliminaries and Definitions}

We begin with the basic definition on harmonic univalent functions.

Definition 2.1. A harmonic, complex-valued, orientation preserving, univalent mapping $f$ defined on $\mathbb{U}$ can be written as:

$$
f=h+\bar{g},
$$

where

$$
h(z)=z+\sum_{k=2}^{\infty} a_{k} z^{k}, \quad g(z)=\sum_{k=1}^{\infty} b_{k} z^{k}, \quad\left|b_{1}\right|<1 .
$$

We call $h$ the analytic part and $g$ the co-analytic part of $f$.

Denote by $\mathcal{S}_{H}(m, n ; \alpha, \beta)$ the class of all functions of the form (1) that satisfy the following inequality:

$$
\sum_{k=2}^{\infty}\left(k^{m}-\alpha k^{n}\right)(1-\beta+\beta k)\left(\left|a_{k}\right|+\left|b_{k}\right|\right) \leq(1-\alpha)\left(1-\left|b_{1}\right|\right)
$$

Where $m \in \mathbb{N}, n \in \mathbb{N}_{0}, m>n, 0 \leq \alpha<1, \beta \geq 0$ and $0 \leq\left|b_{1}\right|<1$.

The class $\mathcal{S}_{H}(m, n ; \alpha, \beta)$ with $\left|b_{1}\right|=0$ will be denoted by $\mathcal{S}_{H}^{0}(m, n ; \alpha, \beta)$.

Definition 2.2. If $h, g$ are the form (2) and if $f=h+\bar{g}, F=H+\bar{G}$, where the functions $H$ and $G$ are given by

$$
H(z)=z+\sum_{k=2}^{\infty} A_{k} z^{k}, \quad G(z)=\sum_{k=1}^{\infty} B_{k} z^{k} .
$$

Then the convolution of $f$ and $F$ is defined to be the function

$$
(f * F)(z)=z+\sum_{k=2}^{\infty} a_{k} A_{k} z^{k}+\overline{\sum_{k=1}^{\infty} b_{k} B_{k} z^{k}},
$$

while the Integral convolution is defined by:

$$
(f \diamond F)(z)=z+\sum_{k=2}^{\infty} \frac{a_{k} A_{k}}{k} z^{k}+\overline{\sum_{k=1}^{\infty} \frac{b_{k} B_{k}}{k} z^{k}} .
$$


SOME DISTORTION THEOREMS FOR NEW SUBCLASS OF HARMONIC ... 3

In Ozturk and Yalcin [9] defined the generalized $\delta$-neighborhood of $\mathrm{f}$ to be the set:

$N(f)=\left\{F: \sum_{k=2}^{\infty}(k-\alpha)\left(\left|a_{k}-A_{k}\right|+\left|b_{k}-B_{k}\right|\right)+(1-\alpha)\left|b_{1}-B_{1}\right| \leq \delta(1-\alpha)\right\}$,

where $F=H+\bar{G}$, and $H, G$ are the form (4).

Theorem 2.3. For harmonic univalent mapping $f$ as above, $\left|a_{1}\right| \leq 2$ and for all $n \geq 2$,

$$
\left|a_{n}\right|=\left|b_{n}\right| \leq 2 / n
$$

Complete proof is explained in theorem 4.7 of [7].

\section{Main Results}

We start this section with the most important following theorem.

Theorem 3.1. For $0 \leq \alpha_{1} \leq \alpha_{2}<1$, we have

$$
\mathcal{S}_{H}\left(m, n ; \alpha_{2}, \beta\right) \subseteq \mathcal{S}_{H}\left(m, n ; \alpha_{1}, \beta\right)
$$

Consequently

$$
\mathcal{S}_{H}^{0}\left(m, n ; \alpha_{2}, \beta\right) \subseteq \mathcal{S}_{H}^{0}\left(m, n ; \alpha_{1}, \beta\right)
$$

In particular

$$
\mathcal{S}_{H}(m, n ; \alpha, \beta) \subseteq \mathcal{S}_{H}(m, n ; 0, \beta)
$$

and

$$
\mathcal{S}_{H}^{0}(m, n ; \alpha, \beta) \subseteq \mathcal{S}_{H}^{0}(m, n ; 0, \beta)
$$

Where $m \in \mathbb{N}, n \in \mathbb{N}_{0}, m>n$ and $\beta \geq 0$.

Proof. Let $f \in \mathcal{S}_{H}\left(m, n ; \alpha_{2}, \beta\right)$, Thus we have

$$
\sum_{k=2}^{\infty} \frac{\left(k^{m}-\alpha_{2} k^{n}\right)(1-\beta+\beta k)}{1-\alpha_{2}}\left(\left|a_{k}\right|+\left|b_{k}\right|\right) \leq 1-\left|b_{1}\right|
$$

Now, using (7), we have

$$
\begin{aligned}
\sum_{k=2}^{\infty} \frac{\left(k^{m}-\alpha_{1} k^{n}\right)(1-\beta+\beta k)}{1-\alpha_{1}}\left(\left|a_{k}\right|+\left|b_{k}\right|\right) & \leq \sum_{k=2}^{\infty} \frac{\left(k^{m}-\alpha_{2} k^{n}\right)(1-\beta+\beta k)}{1-\alpha_{2}}\left(\left|a_{k}\right|+\left|b_{k}\right|\right) \\
& \leq 1-\left|b_{1}\right|
\end{aligned}
$$

This completes the proof of Theorem 3.1.

Theorem 3.2. $\mathcal{S}_{H}(m, n ; \alpha, \beta) \subseteq \mathcal{S}_{H}(\alpha)$ for $m \in \mathbb{N}, n \in \mathbb{N}_{0}, m>n, 0 \leq \alpha<$ $1, \beta \geq 0$ and $\mathcal{S}_{H}(m, n ; \alpha, \beta) \subseteq \mathcal{C}_{H}(\alpha)$ if $\beta \geq 1$ or $m \geq 2, n \geq 1$ and $m>n$. 
4 SOME DISTORTION THEOREMS FOR NEW SUBCLASS OF HARMONIC ...

Proof. Let $f \in \mathcal{S}_{H}(m, n ; \alpha, \beta)$, Then

$$
\sum_{k=2}^{\infty} \frac{\left(k^{m}-\alpha k^{n}\right)(1-\beta+\beta k)}{1-\alpha}\left(\left|a_{k}\right|+\left|b_{k}\right|\right) \leq 1-\left|b_{1}\right|
$$

Now, using (8), we obtain

$$
\sum_{k=2}^{\infty} \frac{k-\alpha}{1-\alpha}\left(\left|a_{k}\right|+\left|b_{k}\right|\right) \leq \sum_{k=2}^{\infty} \frac{\left(k^{m}-\alpha k^{n}\right)(1-\beta+\beta k)}{1-\alpha}\left(\left|a_{k}\right|+\left|b_{k}\right|\right) \leq 1-\left|b_{1}\right|,
$$

Thus, $f \in \mathcal{S}_{H}(\alpha)$ and we get $\mathcal{S}_{H}(m, n ; \alpha, \beta) \subseteq \mathcal{S}_{H}(\alpha)$.

We have to show that $\mathcal{S}_{H}(m, n ; \alpha, \beta) \subseteq \mathcal{C}_{H}(\alpha)$. By using the inequality (8), we have

$\sum_{k=2}^{\infty} \frac{k(k-\alpha)}{1-\alpha}\left(\left|a_{k}\right|+\left|b_{k}\right|\right) \leq \sum_{k=2}^{\infty} \frac{\left(k^{m}-\alpha k^{n}\right)(1-\beta+\beta k)}{1-\alpha}\left(\left|a_{k}\right|+\left|b_{k}\right|\right) \leq 1-\left|b_{1}\right|$, where $\beta \geq 1$ or $m \geq 2, n \geq 1$ and $m>n$.

Thus, $f \in \mathcal{C}_{H}(\alpha)$ and we get $\mathcal{S}_{H}(m, n ; \alpha, \beta) \subseteq \mathcal{C}_{H}(\alpha)$.

Theorem 3.3. The class $\mathcal{S}_{H}(m, n ; \alpha, \beta)$ consists of univalent sense preserving harmonic mappings.

Proof. If $z_{1} \neq z_{2}$ then

$$
\begin{aligned}
\left|\frac{f\left(z_{1}\right)-f\left(z_{2}\right)}{h\left(z_{1}\right)-h\left(z_{2}\right)}\right| & \geq 1-\left|\frac{g\left(z_{1}\right)-g\left(z_{2}\right)}{h\left(z_{1}\right)-h\left(z_{2}\right)}\right| \\
& =1-\left|\frac{\sum_{k=1}^{\infty} b_{k}\left(z_{1}^{k}-z_{2}^{k}\right)}{\left(z_{1}-z_{2}\right)+\sum_{k=2}^{\infty} a_{k}\left(z_{1}^{k}-z_{2}^{k}\right)}\right| \\
& >1-\left|\frac{\sum_{k=1}^{\infty} k\left|b_{k}\right|}{1-\sum_{k=2}^{\infty} k\left|a_{k}\right|}\right| \geq 1-\frac{\sum_{k=1}^{\infty} \frac{\left(k^{m}-\alpha k^{n}\right)(1-\beta+\beta k)}{1-\sum_{k=2}^{\infty} \frac{\left(k^{m}-\alpha k^{n}\right)(1-\beta+\beta k)}{1-\alpha}\left|a_{k}\right|} \geq 0 .}{1-\alpha} \mid
\end{aligned}
$$

which proves univalence. Note that $f$ is sense preserving in $\mathbb{U}$ because

$$
\begin{aligned}
\left|h^{\prime}(z)\right| & \geq 1-\sum_{k=2}^{\infty} k\left|a_{k}\right||z|^{k}>1-\sum_{k=2}^{\infty} \frac{\left(k^{m}-\alpha k^{n}\right)(1-\beta+\beta k)}{1-\alpha}\left|a_{k}\right| \\
& \geq \sum_{k=2}^{\infty} \frac{\left(k^{m}-\alpha k^{n}\right)(1-\beta+\beta k)}{1-\alpha}\left|b_{k}\right|>\sum_{k=2}^{\infty} \frac{\left(k^{m}-\alpha k^{n}\right)(1-\beta+\beta k)}{1-\alpha}\left|b_{k}\right||z|^{k-1} \\
& \geq \sum_{k=1}^{\infty} k\left|b_{k}\right||z|^{k-1} \geq\left|g^{\prime}(z)\right| .
\end{aligned}
$$

So, the proof of Theorem 3.3 is completed. 
SOME DISTORTION THEOREMS FOR NEW SUBCLASS OF HARMONIC ... 5

Theorem 3.4. If $f \in \mathcal{S}_{H}(m, n ; \alpha, \beta)$, then

$$
\left(1-\left|b_{1}\right|\right)\left(|z|-\psi|z|^{2}\right) \leq|f(z)| \leq\left(1+\left|b_{1}\right|\right)|z|+\psi\left(1-\left|b_{1}\right|\right)|z|^{2} .
$$

Where $\psi=\frac{1-\alpha}{(1+\beta)\left(2^{m}-2^{n} \alpha\right)}$, and equalities are attained by the functions:

$$
f_{\theta}(z)=z+\left|b_{1}\right| e^{i \theta} \bar{z}+\left(1-\left|b_{1}\right|\right) \psi z^{2}
$$

and

$$
f_{\theta}(z)=z+\left|b_{1}\right| e^{i \theta} \bar{z}+\left(1-\left|b_{1}\right|\right) \psi \bar{z}^{2}
$$

For properly chosen real $\theta$.

Proof. We have

$$
\begin{aligned}
|f(z)| & \leq\left(1+\left|b_{1}\right|\right)|z|+|z|^{2} \sum_{k=2}^{\infty}\left(\left|a_{k}\right|+\left|b_{k}\right|\right) \\
& \leq\left(1+\left|b_{1}\right|\right)|z|+|z|^{2} \frac{1-\alpha}{(1+\beta)\left(2^{m}-2^{n} \alpha\right)} \sum_{k=2}^{\infty} \frac{(1-\beta+\beta k)\left(k^{m}-k^{n} \alpha\right)}{1-\alpha}\left(\left|a_{k}\right|+\left|b_{k}\right|\right) \\
& \leq\left(1+\left|b_{1}\right|\right)|z|+|z|^{2} \frac{1-\alpha}{(1+\beta)\left(2^{m}-2^{n} \alpha\right)}\left(1-\left|b_{1}\right|\right) .
\end{aligned}
$$

and

$$
\begin{aligned}
|f(z)| & \geq\left(1-\left|b_{1}\right|\right)|z|-\sum_{k=2}^{\infty}\left(\left|a_{k}\right|+\left|b_{k}\right|\right)|z|^{k} \geq\left(1-\left|b_{1}\right|\right)|z|-|z|^{2} \sum_{k=2}^{\infty}\left(\left|a_{k}\right|+\left|b_{k}\right|\right) \\
& \geq\left(1-\left|b_{1}\right|\right)|z|-|z|^{2} \frac{1-\alpha}{(1+\beta)\left(2^{m}-2^{n} \alpha\right)} \sum_{k=2}^{\infty} \frac{(1-\beta+\beta k)\left(k^{m}-k^{n} \alpha\right)}{1-\alpha}\left(\left|a_{k}\right|+\left|b_{k}\right|\right) \\
& \geq\left(1-\left|b_{1}\right|\right)|z|-|z|^{2} \frac{1-\alpha}{(1+\beta)\left(2^{m}-2^{n} \alpha\right)}\left(1-\left|b_{1}\right|\right) \\
& =\left(1-\left|b_{1}\right|\right)\left(|z|-|z|^{2} \frac{1-\alpha}{(1+\beta)\left(2^{m}-2^{n} \alpha\right)}\right) .
\end{aligned}
$$

We define $\psi:=\frac{1-\alpha}{(1+\beta)\left(2^{m}-2^{n} \alpha\right)}$ in inequalities (12) and (13), and so attained (9). It can be easily seen that the functions $f_{\theta}(z)$ defined by (10) and (11) are extremal for Theorem 3.4.

Thus the class $\mathcal{S}_{H}(m, n ; \alpha, \beta)$ is uniformly bounded, and hence it is normal by Montel's Theorem.

Putting $m=1, n=0$ and $\beta=0$ in Theorem 3.4, we obtain the following result which correct the result of Ozturk and Yalcin [9, Theorem 3.6].

corollary 3.5. If $f \in \mathcal{S}_{H}(\alpha)$, then

$$
\left(1-\left|b_{1}\right|\right)\left(|z|-\frac{1-\alpha}{2-\alpha}|z|^{2}\right) \leq|f(z)| \leq\left(1+\left|b_{1}\right|\right)|z|+\frac{(1-\alpha)\left(1-\left|b_{1}\right|\right)}{2-\alpha}|z|^{2} .
$$


Equalities are attained by the functions:

$$
f_{\theta}(z)=z+\left|b_{1}\right| e^{i \theta} \bar{z}+\frac{(1-\alpha)\left(1-\left|b_{1}\right|\right)}{2-\alpha} z^{2}
$$

and

$$
f_{\theta}(z)=z+\left|b_{1}\right| e^{i \theta} \bar{z}+\frac{(1-\alpha)\left(1-\left|b_{1}\right|\right)}{2-\alpha} \bar{z}^{2}
$$

For properly chosen real $\theta$.

Remark 3.6. The above result is different from that of Ozturk and Yalcin [9, Theorem 3.6]. Also, our result gives a better estimate than that of [9] because

$|f(z)| \leq\left(1+\left|b_{1}\right|\right)|z|+\frac{(1-\alpha)\left(1-\left|b_{1}\right|\right)}{2-\alpha}|z|^{2} \leq\left(1+\left|b_{1}\right|\right)|z|+\frac{\left(1-\alpha^{2}\right)\left(1-\left|b_{1}\right|\right)}{2}|z|^{2}$

and

$$
|f(z)| \geq\left(1-\left|b_{1}\right|\right)\left(|z|-\frac{1-\alpha}{2-\alpha}|z|^{2}\right) \geq\left(1-\left|b_{1}\right|\right)\left(|z|-\frac{1-\alpha^{2}}{2}|z|^{2}\right) .
$$

Although, zturk and Yalcin [9] state that the result is sharp for the function

$$
f_{\theta}(z)=z+\left|b_{1}\right| e^{i \theta} \bar{z}+\frac{\left(1-\alpha^{2}\right)\left(1-\left|b_{1}\right|\right)}{2} \bar{z}^{2}
$$

it can be easily seen that the function $f_{\theta}(z)$ does not satisfy the coefficient condition for the class $f \in \mathcal{S}_{H}(\alpha)$ defined by them. Hence, the function $f_{\theta}(z)$ does not belong to the class $f \in \mathcal{S}_{H}(\alpha)$. Therefore the result of Ozturk and Yalcin [9, Theorem 3.6] is incorrect. The correct result is mentioned in (14) and the result is sharp for functions given by (15) and (16), respectively.

Putting $m=2, n=1$ and $\beta=0$ in Theorem 3.4, we obtain the following result which correct the result of Ozturk and Yalcin [9, Theorem 3.6].

corollary 3.7. If $f \in \mathcal{C}_{H}(\alpha)$, then

$$
\left(1-\left|b_{1}\right|\right)\left(|z|-\frac{1-\alpha}{2(2-\alpha)}|z|^{2}\right) \leq|f(z)| \leq\left(1+\left|b_{1}\right|\right)|z|+\frac{(1-\alpha)\left(1-\left|b_{1}\right|\right)}{2(2-\alpha)}|z|^{2} .
$$

Equalities are attained by the functions:

$$
f_{\theta}(z)=z+\left|b_{1}\right| e^{i \theta} \bar{z}+\frac{(1-\alpha)\left(1-\left|b_{1}\right|\right)}{2(2-\alpha)} z^{2}
$$

and

$$
f_{\theta}(z)=z+\left|b_{1}\right| e^{i \theta} \bar{z}+\frac{(1-\alpha)\left(1-\left|b_{1}\right|\right)}{2(2-\alpha)} \bar{z}^{2}
$$

For properly chosen real $\theta$.

Remark 3.8. This result is different from the result of Ozturk and Yalcin [9, Theorem 3.8], and it can be easily seen that our result gives a better 
SOME DISTORTION THEOREMS FOR NEW SUBCLASS OF HARMONIC ... 7

estimate. Also, it can be easily verified that the sharp result for [9, Theorem $3.8]$ is given by the function

$$
f_{\theta}(z)=z+\left|b_{1}\right| e^{i \theta} \bar{z}+\frac{3-\alpha-2 \alpha}{2 \alpha} \bar{z}^{2}
$$

does not belong to the class $f \in \mathcal{C}_{H}(\alpha)$. Hence the result of Ozturk and Yalcin [9] is incorrect. The correct result is given by corollary 3.7.

Theorem 3.9. Let $f=h+\bar{g}$, where $h$ and $g$ are of the form (2). Then $f \in \mathcal{S}_{H}^{0}(m, n ; \alpha, \beta)$ if and only if

$$
f(z)=\sum_{k=1}^{\infty}\left[x_{k} h_{k}(z)+y_{k} g_{k}(z)\right],
$$

where

$$
h_{1}(z)=z, \quad h_{k}(z)=z+\frac{1-\alpha}{(1+\beta)\left(2^{m}-2^{n} \alpha\right)} z^{k} \quad(k=2,3, \ldots)
$$

and

$$
\begin{aligned}
& g_{k}(z)=z+\frac{1-\alpha}{(1+\beta)\left(2^{m}-2^{n} \alpha\right)} \bar{z}^{k} \quad(k=2,3, \ldots), \\
& x_{k}, y_{k} \geq 0, y_{1}=0, x_{1}=1-\sum_{k=2}^{\infty}\left(x_{k}+y_{k}\right) .
\end{aligned}
$$

In particular, the extreme points of the class $\mathcal{S}_{H}^{0}(m, n ; \alpha, \beta)$ are $\left\{h_{k}\right\}$ and $\left\{g_{k}\right\}$.

Proof. Suppose that

$$
\begin{aligned}
f(z) & =\sum_{k=1}^{\infty}\left(x_{k} h_{k}(z)+y_{k} g_{k}(z)\right) \\
& =z+\sum_{k=2}^{\infty}\left(\frac{1-\alpha}{(1-\beta+\beta z)\left(k^{m}-k^{n} \alpha\right)} x_{k} z^{k}+\frac{1-\alpha}{(1-\beta+\beta k)\left(k^{m}-k^{n} \alpha\right)} y_{k} \bar{z}^{k}\right)
\end{aligned}
$$

Than

$$
\begin{aligned}
& \sum_{k=2}^{\infty} \frac{(1-\beta+\beta z)\left(k^{m}-k^{n} \alpha\right)}{1-\alpha}\left(\frac{1-\alpha}{(1-\beta+\beta z)\left(k^{m}-k^{n} \alpha\right)} x_{k} z^{k}\right. \\
& \left.\quad+\frac{1-\alpha}{(1-\beta+\beta k)\left(k^{m}-k^{n} \alpha\right)} y_{k} \bar{z}^{k}\right)=\sum_{k=2}^{\infty} x_{k}+\sum_{k=2}^{\infty} y_{k}=1-x_{1} \leq 1
\end{aligned}
$$

and so $f \in \operatorname{clcoS}_{H}^{0}(m, n ; \alpha, \beta)$.

conversely, if $f \in \operatorname{clco} \mathcal{S}_{H}^{0}(m, n ; \alpha, \beta)$. Set

$x_{k}=\frac{1-\alpha}{(1-\beta+\beta k)\left(k^{m}-k^{n} \alpha\right)}\left|a_{k}\right|, y_{k}=\frac{1-\alpha}{(1-\beta+\beta k)\left(k^{m}-k^{n} \alpha\right)}\left|b_{k}\right|, \quad(k=2,3,4, \ldots)$. 
Then note that $0 \leq x_{k}, y_{k} \leq 1, \quad(k=2,3,4, \ldots)$. We define $x_{1}=$ $1-\sum_{k=2}^{\infty} x_{k}-\sum_{k=2}^{\infty} y_{k}, x_{1} \geq 0$ and $y_{1}=0$. Consequently, we obtain required representation, since

$$
\begin{aligned}
f(z) & =z+\sum_{k=2}^{\infty}\left(\left|a_{k}\right| z^{k}+\left|b_{k}\right| \bar{z}^{k}\right) \\
& =z+\sum_{k=2}^{\infty}\left(\frac{1-\alpha}{(1+\beta)\left(2^{m}-2^{n} \alpha\right)} x_{k} z^{k}+\frac{1-\alpha}{(1+\beta)\left(2^{m}-2^{n} \alpha\right)} y_{k} \bar{z}^{k}\right) \\
& =z+\sum_{k=2}^{\infty}\left(\left(h_{k}(z)-z\right) x_{k}+\left(g_{k}(z)-z\right) y_{k}\right) \\
& =\left(1-\sum_{k=2}^{\infty} x_{k}-\sum_{k=2}^{\infty} y_{k}\right) z+\sum_{k=2}^{\infty} h_{k}(z) x_{k}+\sum_{k=2}^{\infty} g_{k}(z) y_{k} \\
& =\sum_{k=1}^{\infty}\left[x_{k} h_{k}(z)+y_{k} g_{k}(z)\right] .
\end{aligned}
$$

This completes the proof of Theorem 3.9.

Remark 3.10. (i). Putting $m=1, n=0$ and $\beta=0$ in Theorem 3.9, we obtain the extreme points of the class $\mathcal{S}_{H}^{0}(\alpha)$.

(ii). Putting $m=2, n=1$ and $\beta=0$ in Theorem 3.9, we obtain the extreme points of the class $\mathcal{C}_{H}^{0}(\alpha)$.

Let $\mathcal{K}_{H}^{0}$ denote the class of harmonic univalent functions of the form (1) with $b_{1}=0$ that map $\mathbb{U}$ on to convex domains. It is known $[5$, Theorem 5.10], that the sharp inequalities $\left|A_{k}\right| \leq \frac{k+1}{2},\left|B_{k}\right| \leq \frac{k-1}{2}$ are true. These results will be used in the next theorem.

Theorem 3.11. Suppose that $F(z)=z+\sum_{k=1}^{\infty}\left(A_{k} z^{k}+\overline{B_{k} z^{k}}\right)$ belongs to the class $\mathcal{K}_{H}^{0}$. If $f \in \mathcal{S}_{H}^{0}(m, n ; \alpha, \beta)$, then $f * F \in \mathcal{S}_{H}^{0}(m-1, n-1 ; \alpha, \beta)$ if $n>1$ and $f \diamond F \in \mathcal{S}_{H}^{0}(m, n ; \alpha, \beta)$.

Proof. Since $f \in \mathcal{S}_{H}^{0}(m, n ; \alpha, \beta)$, then

$$
\sum_{k=2}^{\infty}\left(k^{m}-\alpha k^{n}\right)(1-\beta+\beta k)\left(\left|a_{k}\right|+\left|b_{k}\right|\right) \leq(1-\alpha)
$$


SOME DISTORTION THEOREMS FOR NEW SUBCLASS OF HARMONIC ... 9

Using (20), we have

$$
\begin{aligned}
& \sum_{k=2}^{\infty}\left(k^{m-1}-\alpha k^{n-1}\right)(1-\beta+\beta k)\left(\left|a_{k} A_{k}\right|+\left|b_{k} B_{k}\right|\right) \\
& \quad=\sum_{k=2}^{\infty}\left(k^{m}-\alpha k^{n}\right)(1-\beta+\beta k)\left(\left|a_{k}\right|\left|\frac{A_{k}}{k}\right|+\left|b_{k}\right|\left|\frac{B_{k}}{k}\right|\right) \\
& \quad \leq \sum_{k=2}^{\infty}\left(k^{m}-\alpha k^{n}\right)(1-\beta+\beta k)\left(\left|a_{k}\right|\left|\frac{k+1}{2 k}\right|+\left|b_{k}\right|\left|\frac{k-1}{2 k}\right|\right) \\
& \quad \leq \sum_{k=2}^{\infty}\left(k^{m}-\alpha k^{n}\right)(1-\beta+\beta k)\left(\left|a_{k}\right|+\left|b_{k}\right|\right) \leq(1-\alpha) .
\end{aligned}
$$

It fallows that $f * F \in \mathcal{S}_{H}^{0}(m-1, n-1 ; \alpha, \beta)$. Next, aggain using (20), we have

$$
\begin{aligned}
& \sum_{k=2}^{\infty}\left(k^{m}-\alpha k^{n}\right)(1-\beta+\beta k)\left(\left|\frac{a_{k} A_{k}}{k}\right|+\left|\frac{b_{k} B_{k}}{k}\right|\right) \\
& \quad \leq \sum_{k=2}^{\infty}\left(k^{m}-\alpha k^{n}\right)(1-\beta+\beta k)\left(\left|a_{k}\right|\left|\frac{k+1}{2 k}\right|+\left|b_{k}\right|\left|\frac{k-1}{2 k}\right|\right) \\
& \quad \leq \sum_{k=2}^{\infty}\left(k^{m}-\alpha k^{n}\right)(1-\beta+\beta k)\left(\left|a_{k}\right|+\left|b_{k}\right|\right) \leq(1-\alpha) .
\end{aligned}
$$

Thus we have $f \diamond F \in \mathcal{S}_{H}^{0}(m, n ; \alpha, \beta)$. This completes the proof of Theorem 3.11 .

Let $\mathcal{S}$ denote the class of analytic univalent functions of the form $F(z)=$ $z+\sum_{k=2}^{\infty} A_{k} z^{k}$. It is well known that the sharp inequality $\left|A_{k}\right| \leq k$ is true. It is needed in the next theorem.

Theorem 3.12. If $f \in \mathcal{S}_{H}^{0}(m, n ; \alpha, \beta)$ and $F \in \mathcal{S}$, then for $|\zeta| \leq 1, f *(F+$ $\zeta \bar{F}) \in \mathcal{S}_{H}^{0}(m-1, n-1 ; \alpha, \beta)$ if $n>1$.

Proof. Since $f \in \mathcal{S}_{H}^{0}(m, n ; \alpha, \beta)$, then

$$
\sum_{k=2}^{\infty}\left(k^{m}-\alpha k^{n}\right)(1-\beta+\beta k)\left(\left|a_{k}\right|+\left|b_{k}\right|\right) \leq(1-\alpha),
$$

Using (21), we have

$$
\begin{gathered}
\sum_{k=2}^{\infty}\left(k^{m-1}-\alpha k^{n-1}\right)(1-\beta+\beta k)\left(\left|a_{k} A_{k}\right|+\left|\zeta b_{k} \overline{A_{k}}\right|\right) \\
\sum_{k=2}^{\infty}\left(k^{m}-\alpha k^{n}\right)(1-\beta+\beta k)\left(\left|a_{k}\right|+\left|b_{k}\right|\right) \leq(1-\alpha) .
\end{gathered}
$$


10 SOME DISTORTION THEOREMS FOR NEW SUBCLASS OF HARMONIC ...

It follows that $f *(F+\zeta \bar{F}) \in \mathcal{S}_{H}^{0}(m-1, n-1 ; \alpha, \beta)$ if $n>1$.

Let $\mathcal{P}_{H}^{0}$ denote the class of complex and harmonic functions $F$ in $\mathbb{U}, F=$ $H+\bar{G}$ such that $\operatorname{Re}\{F(z)\}>0, z \in \mathbb{U}$ and

$$
H(z)=1+\sum_{k=1}^{\infty} A_{k} z^{k}, G(z)=\sum_{k=2}^{\infty} B_{k} z^{k} .
$$

It is known [8, Theorem 3] that the sharp inequalities $\left|A_{k}\right|<k+1,\left|B_{k}\right|<$ $k-1$ are true.

Theorem 3.13. Suppse that $F(z)=1+\sum_{k=2}^{\infty}\left(A_{k} z^{k}+\overline{B_{k} z^{k}}\right)$ belong to class $\mathcal{P}_{H}^{0}$. If $f \in \mathcal{S}_{H}^{0}(m, n ; \alpha, \beta)$ and for $\frac{2}{3} \leq\left|A_{1}\right| \leq 2$, then $\frac{1}{A_{1}} f * F \in \mathcal{S}_{H}^{0}(m-$ $1, n-1 ; \alpha, \beta)$ if $n \geq 1$ and $\frac{1}{A_{1}} f \diamond F \in \mathcal{S}_{H}^{0}(m, n ; \alpha, \beta)$.

Proof. Since $f \in \mathcal{S}_{H}^{0}(m, n ; \alpha, \beta)$, then

$$
\sum_{k=2}^{\infty}\left(k^{m}-\alpha k^{n}\right)(1-\beta+\beta k)\left(\left|a_{k}\right|+\left|b_{k}\right|\right) \leq(1-\alpha)
$$

Using (22), we have

$$
\begin{aligned}
& \sum_{k=2}^{\infty}\left(k^{m-1}-\alpha k^{n-1}\right)(1-\beta+\beta k)\left(\left|\frac{a_{k} A_{k}}{A_{1}}\right|+\left|\frac{b_{k} B_{k}}{A_{1}}\right|\right) \\
& \leq \sum_{k=2}^{\infty}\left(k^{m}-\alpha k^{n}\right)(1-\beta+\beta k)\left(\left|\frac{a_{k}}{A_{1}}\right| \frac{k+1}{k}+\left|\frac{b_{k}}{A_{1}}\right| \frac{k-1}{k}\right) \\
& \leq \sum_{k=2}^{\infty}\left(k^{m}-\alpha k^{n}\right)(1-\beta+\beta k)\left(\left|a_{k}\right|+\left|b_{k}\right|\right) \leq(1-\alpha) .
\end{aligned}
$$

Thus $\frac{1}{A_{1}} f * F \in \mathcal{S}_{H}^{0}(m-1, n-1 ; \alpha, \beta)$ if $n \geq 1$.

Similarly, we can show that $\frac{1}{A_{1}} f \diamond F \in \mathcal{S}_{H}^{0}(m, n ; \alpha, \beta)$. This completes the proof of Theorem 3.13.

Theorem 3.14. Let

$$
f(z)=z+\overline{b_{1} z}+\sum_{k=2}^{\infty}\left(a_{k} z^{k}+\overline{b_{k} z^{k}}\right)
$$

be a member of the class $\mathcal{S}_{H}(m, n ; \alpha, \beta)$. If

$$
\delta \leq \frac{\left(1-\left|b_{1}\right|\right)\left(\left(2^{m}-2^{n} \alpha\right)(1+\beta)-1\right)}{\left(2^{m}-2^{n} \alpha\right)(1+\beta)}
$$

then $N(f) \subset \mathcal{S}_{H}(\alpha)$. 
Proof. Let $f \in \mathcal{S}_{H}(m, n ; \alpha, \beta)$ and $F(z)=z+\overline{B_{1} z}+\sum_{k=2}^{\infty}\left(A_{k} z^{k}+\overline{B_{k} z^{k}}\right)$ belong to $N(f)$. We have

$$
\begin{aligned}
& (1-\alpha)\left|B_{1}\right|+\sum_{k=2}^{\infty}(k-\alpha)\left(\left|A_{k}\right|+\left|B_{k}\right|\right) \\
& \leq(1-\alpha)\left|B_{1}-b_{1}\right|+\sum_{k=2}^{\infty}(k-\alpha)\left(\left|A_{k}-a_{k}\right|+\left|B_{k}-b_{k}\right|\right) \\
& +(1-\alpha)\left|b_{1}\right|+\sum_{k=2}^{\infty}(k-\alpha)\left(\left|a_{k}\right|+\left|b_{k}\right|\right) \\
& \leq(1-\alpha) \delta+(1-\alpha)\left|b_{1}\right|+\frac{1}{(1+\beta)\left(2^{m}-2^{n} \alpha\right)} \sum_{k=2}^{\infty}(1-\beta+\beta k)\left(k^{m}-k^{n} \alpha\right)\left(\left|a_{k}\right|+\left|b_{k}\right|\right) \\
& \leq(1-\alpha) \delta+(1-\alpha)\left|b_{1}\right|+\frac{(1-\alpha)\left(1-\left|b_{1}\right|\right)}{(1+\beta)\left(2^{m}-2^{n} \alpha\right)} \leq 1-\alpha . \\
& \text { If } \delta \leq \frac{\left(1-\left|b_{1}\right|\right)\left(\left(2^{m}-2^{n} \alpha\right)(1+\beta)-1\right)}{\left(2^{m}-2^{n} \alpha\right)(1+\beta)} \text { Thus } F(z) \in \mathcal{S}_{H}(\alpha) .
\end{aligned}
$$

\section{ACKNOWLEDGMENTS}

The Authors would like to express their thanks to their colleagues, professor Ali Ebadian and Dr. Ahmad Zireh, for their suggestions. This work is partially supported the first author by a grant of The University of Shahrood and the second author by a grant of Young Researchers and Elite Club, Malard Branch, Islamic Azad University.

\section{REFERENCES}

[1] A.G. Alanoush, Subclass of harmonic univalent functions associated with the generalized Mittag-Leffler type functions, arXiv:1901.08454v1 [math.CV] 24 Jan 2019.

[2] A.K. Al-khafaji, W.G. Atshan, S.S. Abed, On the Generalization of a Class of Harmonic Univalent Functions Defined by Differential Operator, 312(6) (2018), $1-9$.

[3] O. Altintas, Ozkan, H.M. Srivastava, Neighborhoods of a class of analytic functions with negative coefficients, Appl. Math. Letters, 13 (2000), 63-67.

[4] Y. Avci, E. Zlotkiewicz, On harmonic univalent mappings,Ann. Univ. Marie Curie-Sklodowska Sect. A, 44 (1990), 1-7.

[5] J. Clunie, T. Sheil-Small, Harmonic univalent functions, Ann. Acad. Sci. Fenn. Ser. A.I, 9 (1984) ,3-25.

[6] K.K. Dixit, S. Porwal, On a subclass of harmonic unnivalent functions, Journal of inequalities in pure and applied mathematics, 10(27) (2009), 1-9.

[7] W. Hengartner, G. Schober, Univalent harmonic functions, Trans. Amer. Math. Soc., 299(1) (1987), 1-31.

[8] Z.J. Jakubowski, W. Majchrzak, K. Skalska, Harmonic mappings with a positive real part, Materialy Konferencji z Teorii Zagadnien Ekstremalnych Lodz, 14 (1993), 17-24. 
12 SOME DISTORTION THEOREMS FOR NEW SUBCLASS OF HARMONIC ...

[9] M. Ozturk, S. Yalcin, On univalent harmonic functions, J. Inequal. Pure Appl. Math., 3(4) (2002), 1-8 .

[10] S. Ruscheweyh, Neighborhoods of univalent functions, Proc. Amer. Math. Soc. 81 (1981), 521-528.

[11] T.M. Seoudy, M.K. Aouf, Several properties of certain classes of univalent harmonic functions, Afr. Mat., 26 (2014), 627-636.

[12] T.M. Seoudy, Some Properties Of Certain Classes Of Harmonic Univalent Functions, AL.I. CUZA, (2013), 1-10 . 